\title{
Neurogenic Stunned Myocardium with Ventriculoperitoneal Shunt Malfunction
}

\author{
Ron Levy, Walter J. Hader
}

Can. J. Neurol. Sci. 2011; 38: 518-521

The syndrome of left ventricular dysfunction in patients without obstructive coronary artery disease following central nervous system injury is termed neurogenic stunned myocardium (NSM). It has been described most commonly in association with aneurysmal subarachnoid hemorrhage. Nonspecific electrocardiogram (ECG) abnormalities, elevated troponin I levels, and reversible regional-wall motion abnormalities on echocardiogram are observed ${ }^{1,2}$. In patients with subarachnoid hemorrhage-related NSM, angiography demonstrates normal coronary arteries and a transient left ventricular dysfunction ${ }^{2}$. A wide variety of neurologic etiologies may also give rise to NSM. These include but are not limited to intracerebral hemorrhage, ischemic stroke, traumatic brain injury, cerebral infarction, seizures, and metastatic brain tumors.
There is a paucity of reports of hydrocephalus related NSM given the widespread use of cerebral spinal fluid shunts in the neurosurgical patient population. We report a case of NSM in a shunt dependant patient with acute hydrocephalus secondary to a shunt malfunction.

\section{CASE REPORT}

A 53-year-old woman had a witnessed collapse at home that was heralded by complaints of sudden onset chest pain and a severe headache. Her past medical history was remarkable for a ventriculoperitoneal shunt inserted 15 years ago to treat obstructive hydrocephalus secondary to a benign tectal region mass. She also suffered from chronic obstructive lung disease

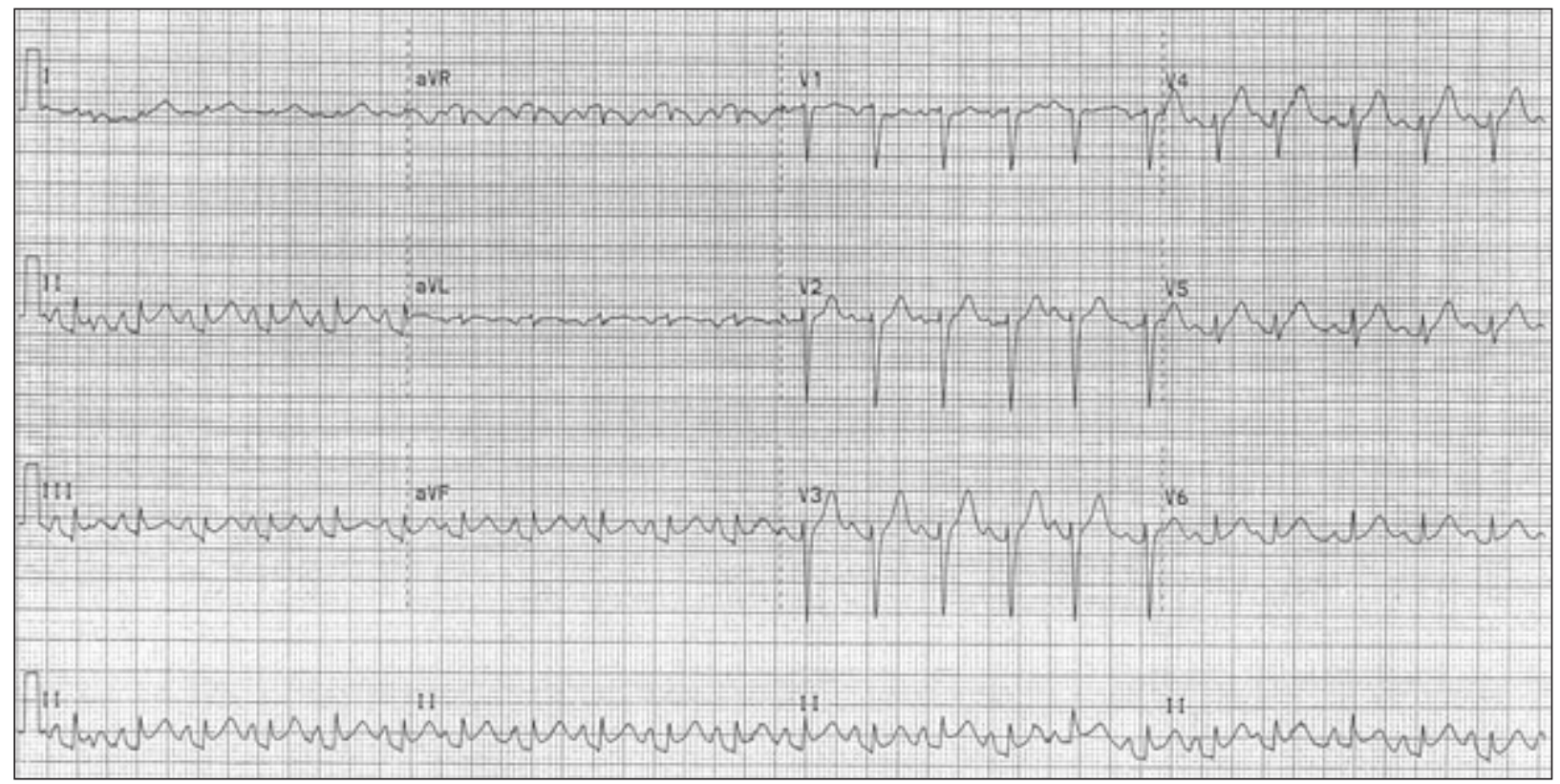

Figure 1: Electrocardiogram demonstrated ST segment elevation in the inferiolateral leads (II, III, AvF).

From the Division of Neurosurgery, Department of Clinical Neuroscience, Faculty of Medicine, University of Calgary, Foothills Medical Centre, Calgary, Alberta, Canada. Received October 22, 2010. Final Revisions Submitted January 10, 2011.

Correspondence to: Walter J. Hader, Division of Neurosurgery, Department of Clinical Neurosciences, Foothills Medical Centre, 12th floor, 1403-29th Street NW, Calgary, Alberta, T2N 2T9, Canada. 


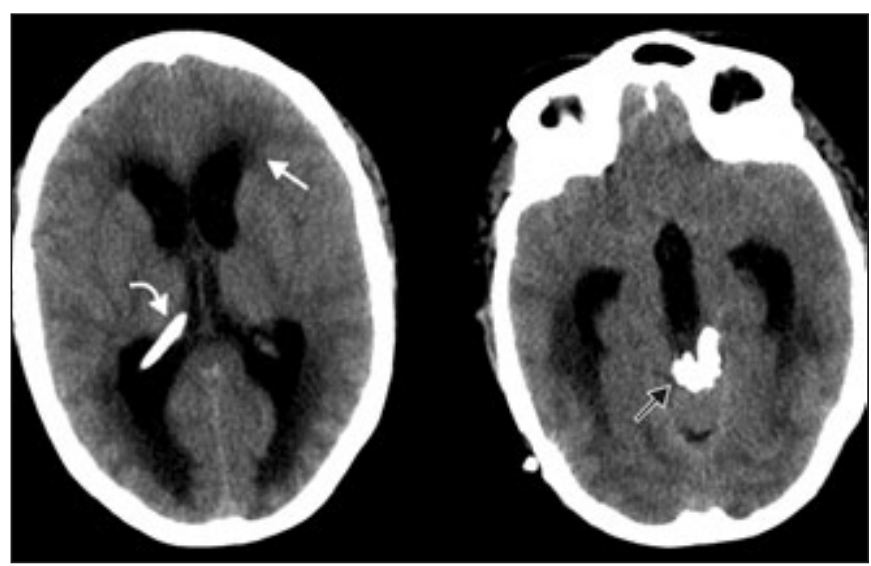

Figure 2: Axial CT scan of the brain shows dilated lateral ventricles (left panel) and third ventricle and temporal horns (right panel). Transependymal flow around the frontal horns (left panel, white arrow), the tip of the shunt catheter (left panel, curved white arrow), and the calcified tectal lesion (right panel, black arrow) are demonstrated.

requiring home oxygen, hypertension, depression, and previous alcohol abuse. There was no prior history of coronary artery disease. Her medications were Ventolin, Atrovent, Avalide, Wellbutrin, Imipramine, Effexor, and Tryptophan. She smoked cigarettes and had no allergies.

On presentation to a peripheral hospital emergency department the patient was confused, agitated, and dyspneic. Apart from a leukocytosis $\left(15.2 \times 10^{3}\right)$ and an elevated creatine

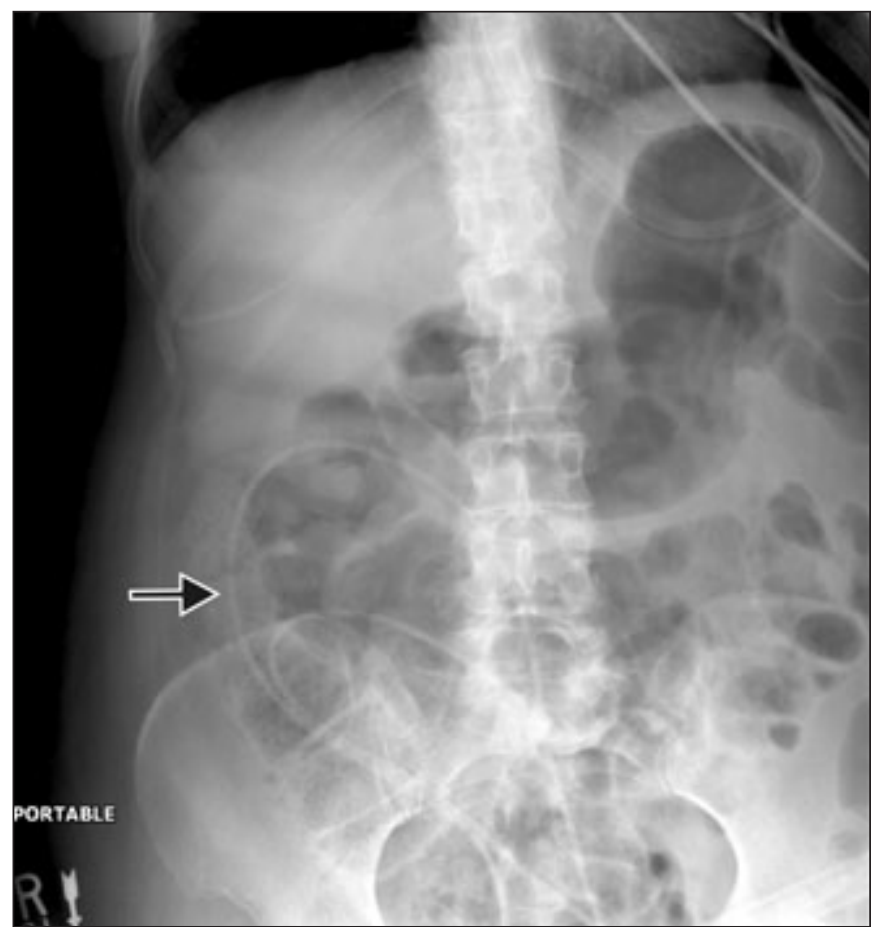

Figure 3: An anteroposterior abdominal radiograph demonstrates a broken and coiled peritoneal catheter (black arrow). kinase $(395 \mathrm{U} / \mathrm{L}$, normal range $=0-150 \mathrm{U} / \mathrm{L})$, serum blood work including troponin I and toxicology was normal. A chest radiograph demonstrated hyperinflation. The patient was admitted for physiologic support related to the exacerbation of chronic obstructive pulmonary disease together with possible alcohol withdrawal and serotonin syndrome. The patient was stabilized with hydration, Solumedrol, and withdrawal of psychotropic medications. After 48 hours she became acutely dyspneic with a declining level of consciousness that required intubation. An arterial blood gas indicated hypercarbic respiratory failure. An ECG displayed ST segment elevation in the inferiolateral leads (II, III, AvF) (Figure 1). These changes were transient in nature and markedly improved with intravenous nitroglycerin therapy. Blood work revealed an elevation in both creatine kinase (1751 U/L) and troponin I (7.11

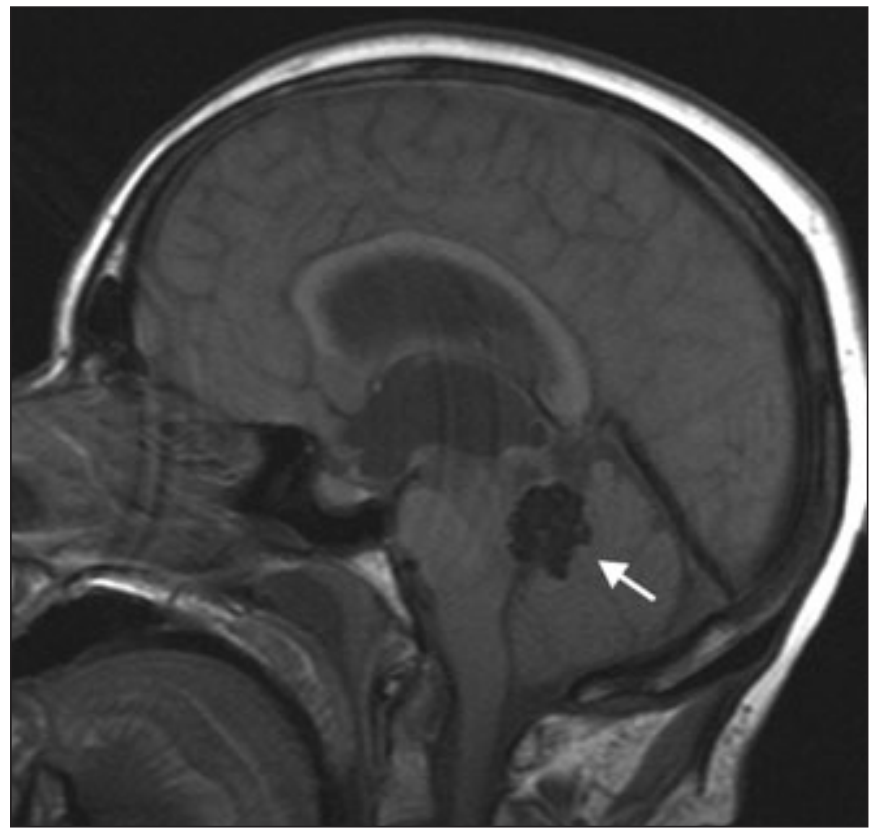

Figure 4: A sagittal T1 weighted MRI shows aqueductal obstruction secondary to a tectal region mass (white arrow).

ug $/ \mathrm{L}$, normal range $=0-0.1 \mathrm{ug} / \mathrm{L})$. An ECG demonstrated left ventricular apical akinesis but with a normal ejection fraction. The patient was extubated within 24 hours and was transferred to our facility with the diagnosis of ST elevation myocardial infarction. A brain computed tomogram (CT) scan had been performed but was interpreted only as being abnormal prior to transfer. It demonstrated dilation of the lateral and third ventricles and compression of cortical sulci consistent with obstructive hydrocephalus secondary to shunt malfunction.

En route the patient's level of consciousness deteriorated and she was reintubated. Her glascow coma scale was 7 T. She was assessed by the cardiology service and was noted to have normal cardiovascular function and no signs of congestive heart failure. A repeat CT showed clearly enlarged third and lateral ventricles 


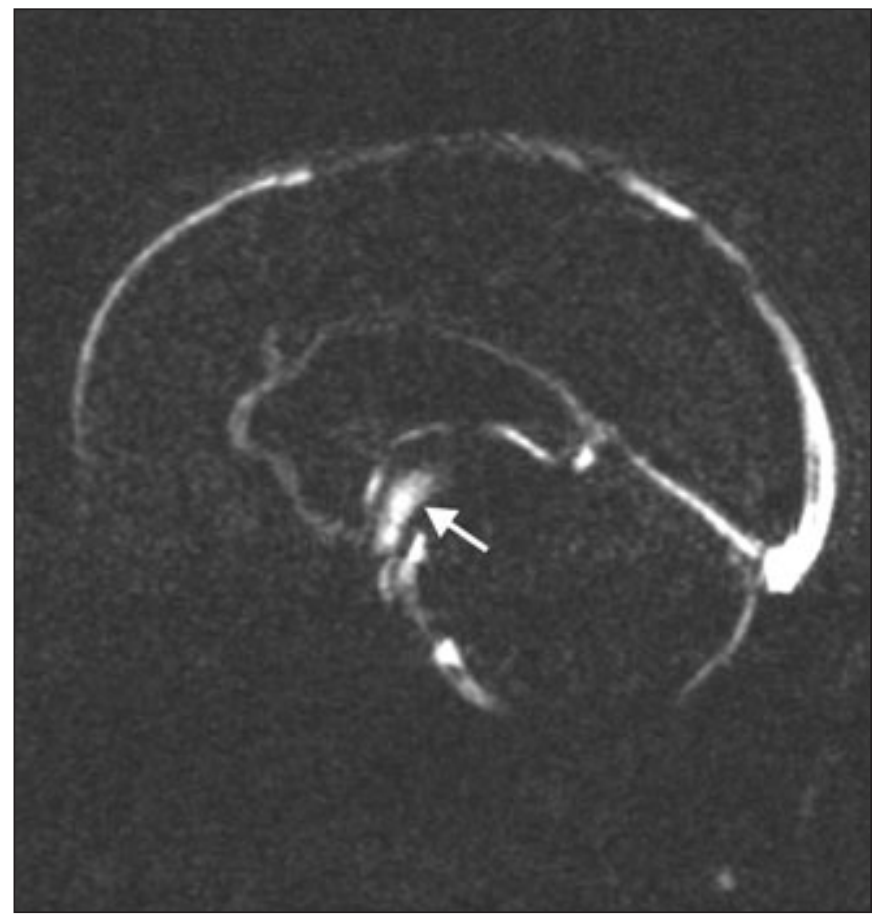

Figure 5: Sagittal cine MRI showing pulsatile flow of cerebrospinal fluid through the third ventriculostomy (white arrow).

and significant transependymal edema (Figure 2) consistent with acute hydrocephalus. A calcified tectal region lesion was observed. A shunt reservoir tap was performed at the bedside and $20 \mathrm{cc}$ of clear cerebrospinal fluid (CSF) was aspirated. Following this maneuver the patient immediately roused and was able to follow commands. A plain radiograph series of the shunt showed that the peritoneal catheter was not present over the chest and was coiled in the peritoneal space (Figure 3). A coronary angiogram was performed that demonstrated angiographically normal coronary arteries. An external ventricular drain was inserted. An magnetic resoance image (MRI) the following day clearly demonstrated aqueductal obstruction due to the tectal region lesion (Figure 4). The patient underwent an endoscopic third ventriculostomy. The ventriculoperitoneal shunt was removed and was confirmed broken distal to the shunt valve along the peritoneal catheter. A post-operative cine MRI demonstrated pulsatile flow of CSF through the third ventriculostomy (Figure 5) and a concordant decrease in the degree of hydrocephalus (Figure 6). Her post-operative course was unremarkable and she remained alert and able to follow commands. The patient had no further cardiac problems. She was transferred back to her peripheral hospital after three days. A brain MRI with cine sequences was performed at follow up one month after discharge. It demonstrated a patent third ventriculostomy with decreased ventricular size. There were no cardiac issues noted in the clinical history at that time.

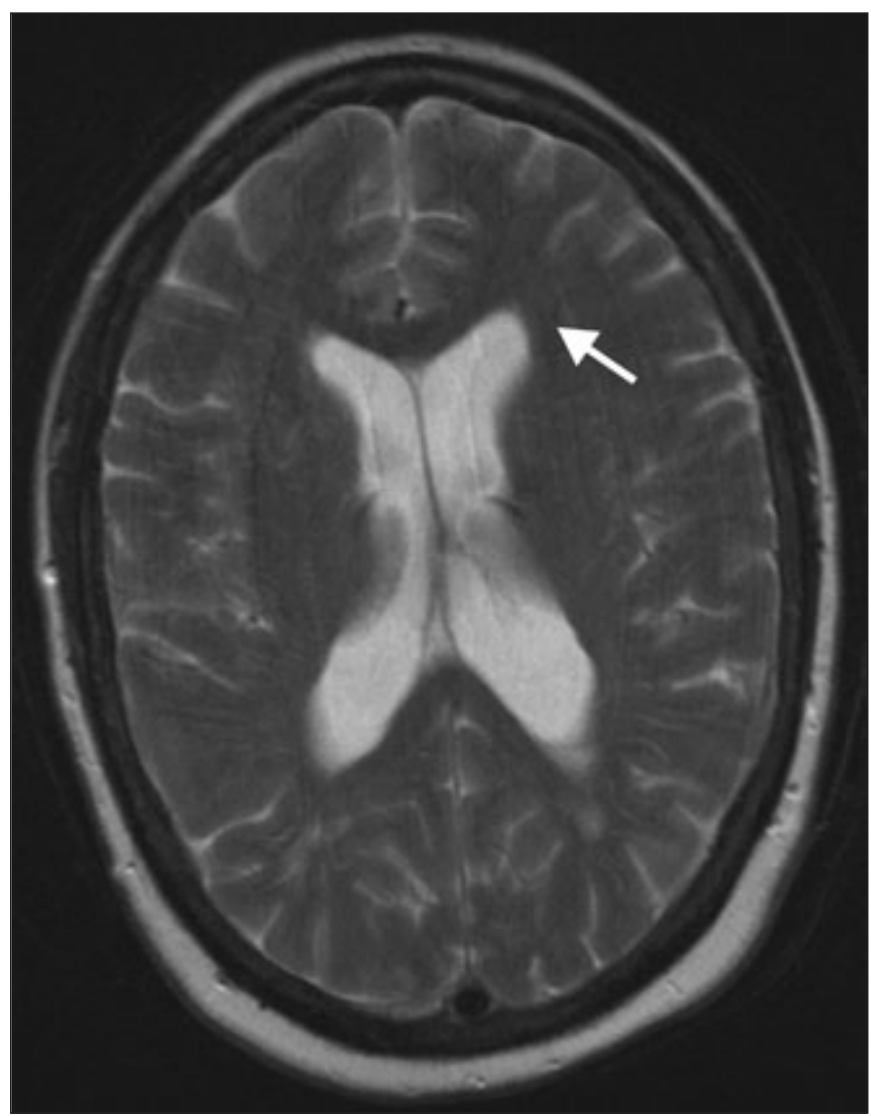

Figure 6: Axial T2 weighted MRI demonstrating an absence of T2 hyperintensity around the frontal horns consistent with a decrease in transependymal flow (white arrow) following the endoscopic third ventriculostomy.

\section{Discussion}

We present a case of a patient with clinical features and cardiac investigations that are suggestive of NSM which was precipitated by a ventriculoperitoneal shunt malfunction. The patient's reduced level of consciousness was related to acute hydrocephalus that was amenable to shunt tapping. With regard to elevated intracranial pressure, the association of NSM due to acute hydrocephalus has been associated with obstructive mass lesions. These include a third ventricular colloid cyst ${ }^{3}$ in an adult and a posterior fossa mass and a tectal glioma in two pediatric patients ${ }^{4}$. Neurogenic stunned myocardium has been documented in a patient with hemorrhagic brain contusion and cerebral edema whose cardiac function resolved following a decompressive craniotomy ${ }^{5}$. Similarly, the mechanism of NSM in our patient was likely due to acute elevation of intracranial pressure.

The pathogenesis of NSM has been hypothesized to be due to excessive sympathetic nervous system activation ${ }^{1}$. The transient left ventricular dysfunction described with NSM may resemble an acute myocardial infarct with non-specific ECG changes and cardiac marker derangements ${ }^{6}$. Echocardiograms in NSM show 
regional wall motion abnormalities such as apical sparing that are not consistent with the patterns observed in acute coronary syndrome $^{2}$. In our patient echocardiography demonstrated apical hypokinesis. We did not observe left mid-ventricular wallmotion abnormalities and apical "ballooning" that specifically characterizes tako-tsubo cardiomyopathy ${ }^{6}$. It has been noted that the cardiac dysfunction observed in subarachnoid hemorrhage related NSM overlaps significantly with tako-tsubo cardiomyopathy and that both syndromes likely share the same pathogenesis $^{1}$. Interestingly, tako-tsubo cardiomyopathy has been observed to predominately occur in postmenopausal women ${ }^{6}$. It has been proposed that sudden death in acute CSF obstruction may be related to acute neurogenic cardiac dysfunction rather than acute hydrocephalus with brain herniation and respiratory compromise ${ }^{3}$. Similarly, myocardial injury may potentiate death in acute hydrocephalus secondary to shunt failure. In patients with severe hydrocephalus, NSM may result in reduction of cardiac output and the risk of cerebral hypoperfusion similar to that observed following high grade subarachnoid hemorrhage.

The clinical presentation of a shunt malfunction is typically related to the acuity of the rise of intracranial hypertension. In patients that are shunt dependent, a malfunction leads to a more precipitant decline and may result in neurological injury if treatment is delayed. The initial symptoms of shunt malfunction are nausea, vomiting, and headache, and may progress to include an altered mental status marked by lethargy, irritability, and drowsiness. Rather than replace the shunt an endoscopic third ventriculostomy was performed. This procedure as an alternative to shunt revision has resulted in shunt independence in approximately $80 \%$ of patients with obstructive hydrocephalus ${ }^{7}$.

Although our patient complained of chest pain and headache at presentation, her cardiac abnormalities became apparent after two days in hospital and coincided with a decline in her level of consciousness. It has been noted that NSM may occur immediately or be delayed following aneurysm rupture ${ }^{1}$. The delay in the decline of her level of consciousness may have been due to compensated hydrocephalus or the marginal flow of CSF through a tenuous subcutaneous fibrous tract left by the broken peritoneal catheter. It is interesting to note that the brain CT performed at the peripheral hospital was initially interpreted as being normal. Computed tomogram scans of shunt dependant patients with hydrocephalus may show no change or minimal change but still result in symptoms due to decreased ventricular compliance.

This case example highlights the need to consider the diagnosis of NSM in patients presenting with myocardial dysfunction and a history of shunt dependant obstructive hydrocephalus. Until otherwise proven a decline in level of consciousness should be attributed to shunt malfunction and treatment should be directed at ruling out acute hydrocephalus. With treatment of the underlying neurologic precipitant, patients usually go on to a full cardiac recovery ${ }^{1,3,4}$.

\section{REFERENCES}

1. Lee VH, Oh JK, Mulvagh SL, Wijdicks EF. Mechanisms in neurogenic stress cardiomyopathy after aneurysmal subarachnoid hemorrhage. Neurocrit Care. 2006;5(3):243-9.

2. Zaroff JG, Rordorf GA, Ogilvy CS, Picard MH. Regional patterns of left ventricular systolic dysfunction after subarachnoid hemorrhage: evidence for neurally mediated cardiac injury. J Am Soc Echocardiogr. 2000;13(8):774-9.

3. Jarquin-Valdivia AA, Rich AT, Yarbrough JL, Thompson RC. Intraventricular colloid cyst, hydrocephalus and neurogenic stunned myocardium. Clin Neurol Neurosurg. 2005;107(5): 361-5.

4. Johnson J, Ragheb J, Garg R, Patten W, Sandberg DI, Bhatia S. Neurogenic stunned myocardium after acute hydrocephalus. J Neurosurg Pediatr. 2010;5(5):428-33.

5. Deleu D, Kettern MA, Hanssens Y, Kumar S, Salim K, Miyares F. Neurogenic stunned myocardium following hemorrhagic cerebral contusion. Saudi Med J. 2007;28(2):283-5.

6. Bybee KA, Kara T, Prasad A, et al. Systematic review: transient left ventricular apical ballooning: a syndrome that mimics STsegment elevation myocardial infarction. Ann Intern Med. 2004;141(11):858-65.

7. Hader WJ, Walker RL, Myles ST, Hamilton M. Complications of endoscopic third ventriculostomy in previously shunted patients. Neurosurgery. 2008;63 Suppl 1:168-74. 